\title{
Compact Monothetic Semirings
}

\author{
Boris Tanana \\ Higher Institute for Science and Technology of Mozambique (ISCTEM), Maputo, Mozambique \\ e-mail: btanana@mail.ru
}

Abstract - In this article we present a brief history and some applications of semirings, the structure of compact monothetic semirings. The classification of these semirings be based on known description of discrete cyclic semirings and compact monothetic semigroups.

Keywords - semigroup, semiring, topological semiring, cyclic semiring, monothetic semigroup, compact monothetic semigroup, compact monothetic semiring.

\section{INTRODUCTION}

A semiring is an algebraic structure in which we can add and multiply elements, where multiplication distributes over addition.

The most familiar examples for semirings in classical algebra are the semiring of non-negative integers or the semiring of nonnegative real numbers with ordinary operations of addition and multiplication. The first examples of semirings appeared in the work of Dedekind [1]. But, then, it was the American mathematician Vandiver who used the term "semi-ring" in his 1934 paper for introducing an algebraic structure with two operations of addition and multiplication such that multiplication distributes on addition [2]

One of the classic areas of mathematics in which semirings arise is the theory of formal languages. A formal language is any set of words (finite strings of symbols) taken from a fixed finite set, called the alphabet. The set of all languages from a fixed alphabet can be viewed as a semiring where addition is the union of the languages and multiplication is the concatenation of the languages. Such semirings have applications in logic and theoretical computer science.

As a subset of all languages the set of regular languages is closed under union and concatenation, so is a semiring. By the famous result of Kleene, the regular languages are precisely the languages accepted by automata [3]. The operation of an automaton can be simulated by repeatedly multiplying the Boolean matrices that encode its rules [4]. This means that the study of regular languages boils down to considering finite collections of matrices over the Boolean semiring $\{0,1\}$ where addition is maximum and multiplication is minimum.
Tropical algebra is a relatively new area of mathematics which brings together ideas from algebra, order theory and discrete mathematics, and which has numerous applications in scheduling and optimization, formal language theory, numerical analysis and dynamical systems. Here the primary objects of study are the tropical semirings. The tropical semirings based on either the non-negative integer, the integer or real numbers, but with unusual semiring operations. Specifically, addition is either maximum (or minimum) and multiplication is usual addition.

Let's move to studying of compact monothetic semirings. We give, initially, the necessary definitions, notions and designations.

\section{BASIC DEFINITIONS AND PRELIMINARY RESULTS}

Definition 1. Semiring is an algebraic structure $\langle S,+, *\rangle$ where $\mathrm{S}$ is a set, $\langle S,+\rangle$ and $\langle S, *\rangle$ are semigroups (i.e., both operations, addition " + "” and multiplication " * ", are associative) and those operations satisfy the laws of distributivity for both sides:

$$
\begin{gathered}
a *(b+c)=a * b+a * c \text { and }(a+b) * c=a * c+b * \\
c, \text { for any } a, b, c \in S .
\end{gathered}
$$

The concept of semiring in this form was introduced by Vandiver in 1934 [2]. Sometimes we will suppose that a semiring $S$ has the neutral element 0 (zero) relatively the addition or/and has the neutral element 1 (unit) relatively the multiplication and $0 * x=x * 0=0$ for any $x \in S$.

Definition 2. If $\langle S, *\rangle$ is a group then a semiring $<S$, ,$+ *>$ is named semifield.

Definition 3. Semigroup $S$ is named cyclic (monogenic) semigroup if it is generated by an element $a \in S$ (i.e, $\left.S=\left\{a, a^{2}, \cdots, a^{n}, \cdots\right\}\right)$ and this fact is denoted by $S=(a)$. 
There exist two cases:

i) $\quad S$ is an infinite.

ii) $\quad S$ is a finite $S=\left\{a, a^{2}, \cdots, a^{k}, \cdots, a^{k+n-1}\right\}$ where $\mathrm{a}^{\mathrm{k}}=\mathrm{a}^{\mathrm{k}+\mathrm{n}}$. In this case $S$ is named the cyclic semigroup of type $(k, n)$.

Definition 4. Semiring $\langle S,+, *\rangle$ is named (multiplicatively) cyclic semiring if $\langle S, *\rangle$ is a cyclic semigroup. If $\langle S, *\rangle$ is generated by element $a \in S$ we will write $S=(a)$. If $S$ has a unit 1 then $S=\left\{a^{n} \mid n \in N_{0}\right\}$ and $a^{0}=1$.

The semiring $\mathrm{S}$ with multiplicative semigroup of type $(k, n)$ is named the cyclic semiring of type $(k, n)$.

Cyclic semifield $\left\{a^{k}, \cdots, a^{k+n-1}\right\}$ is named cycle of the cyclic semiring of type $(k, n)$.

In this work we will consider only multiplicatively cyclic semirings. Thus, further we will omit the term multiplicatively.

So, any finite cyclic semigroup that is not a group has the form:

$S=\left\{a, a^{2}, \cdots, a^{k}, a^{k+1}, \cdots, a^{k+n}\right\}$,

$a^{k+n+1}=a^{k+1}$

or, $T=\left\{1, a, \cdots, a^{k}, a^{k+1}, \cdots, a^{k+n-1}\right\}, k, n \in N$ and $a^{k+n}=a^{k}$.

If we set on multiplicative semigroups $\mathrm{S}$ and $\mathrm{T}$, all operations of addition transform $\mathrm{S}$ and $\mathrm{T}$ into a semiring and we will get all finite cyclic semirings of the case (1) or the case (2).

ijection $a^{m} \leftrightarrow a^{m-1}, m \in N$, between semirings (1) and (2) transfer the operation of addition of (1) to (2) and viceversa.

The semirings obtained from (1) and (2), $\mathrm{S}$ and $\mathrm{T}$ has isomorphs cyclic semifields $\left\{a^{k+1}, a^{k+2}, \cdots, a^{k+n}\right\}$ and $\left\{a^{k}, a^{k+1}, \cdots, a^{k+n-1}\right\}$ respectively.

Theorem 1. ([5]). Between a class of all finite cyclic semirings without unit and a class of all finite cyclic semirings with unit, exist a natural bijection.

Later on we will consider semirings without unit.

Definition 5. A semiring $S$ where $\langle S,+>$ is an idempotent semigroup (i.e., $\quad x+x=x$ for any $x \in S$ ) is called idempotent semiring.
The operation of addition, called left (or right) if $x+y=x$ (or $x+y=y$ ) for any $x, y \in S$.

If $\langle S,+\rangle$ is a commutative idempotent semigroup then $<$ $S,+>$ becomes upper semilattice under the relation of order $x \leq y \Leftrightarrow x+y=y$ for any $x, y \in S$.

Let $S$ be a cyclic semiring $S=(a)$. There, is easy to identify that $\mathrm{S}$ is an idempotent semiring if $a+a=a$ (or $1+1=1$, if $S$ has unit).

Now we will need to give the description of the structure of discrete infinite cyclic semirings received by Vechtomov [6], [7].

\section{Theorem 2.}

i) Infinite cyclic semirings with commutative addition are isomorphic one of the following two cases:

a) $(N, \max ,+)$;

b) $(N, \min ,+)$.

ii) In the infinite cyclic semirings with noncommutative addition, the addition is idempotent and is either left or right.

Definition 6. Let $S$ be a topological space and $X \subseteq S$, by $\bar{X}$ we will denote a closure of subset $X$ in a topological space $\mathrm{S}$. If $\mathrm{S}$ is a semigroup and Hausdorf topological space where an operation is continuous then $\mathrm{S}$ is a topological semigroup. A topological semigroup $S$ is said to be a monothetic semigroup if $\mathrm{S}$ is a closure of a cyclic semigroup (i.e., $S=\overline{(a)}$ for some element $a \in S$ ).

From here, we will, now, reduce the description of the structure of a compact monothetic semigroups belonging to E. Hewitt.

Theorem 3 [8]. A compact monothetic semigroup S has one of the following three characteristics:

i) $\mathrm{S}$ is a compact monothetic group $S=$ $\overline{\left\{a, a^{2}, \cdots, a^{n}, \cdots\right\}}$;

ii) $\mathrm{S}$ contains a compact monothetic group $H=\overline{\left\{b, b^{2}, \cdots, b^{n}, \cdots\right\}}$ with generating element $\mathrm{b}$, unit element e of $\mathrm{H}$ and a finite number of distinct discreet elements $a, a^{2}, \cdots, a^{s}$ which don't belong to $H, S=H \cup\left\{a, a^{2}, \cdots, a^{s}\right\}$, $a e=b$ and, 


$$
\left\{\begin{array}{c}
a^{i} \cdot a^{j}=a^{i+j} ; \text { if } i+j \leq s \\
a^{i} \cdot a^{j}=b^{i+j} ; \text { if } i+j>s \\
a^{i} \cdot x=b^{i} x ; \text { if } x \in H, i=1,2, \cdots, s \\
x \cdot a^{i}=x \cdot b^{i} ; \text { if } x \in H, i=1,2, \cdots, s
\end{array}\right.
$$

iii)

S contains a compact monothetic group $H=\overline{\left\{b, b^{2}, \cdots, b^{n}, \cdots\right\}}$ with generating element $\mathrm{b}$, unit element $\mathrm{e}$ of $\mathrm{H}$, and infinite number of distinct discreet elements $a, a^{2}, \cdots, a^{s}, \cdots$ which don't belong to $H, S=H \cup$ $\left\{a, a^{2}, \cdots, a^{n} \cdots\right\}, a e=b$

$$
\left\{\begin{array}{c}
a^{i} \cdot a^{j}=a^{i+j} \\
a^{i} \cdot x=b^{i} x ; \text { if } x \in H, i=1,2, \cdots \\
x \cdot a^{i}=x \cdot b^{i} ; \text { if } x \in H, i=1,2, \cdots
\end{array}\right.
$$

Let $x \in H$ and $V(x)$ any neighborhood of $\mathrm{x}$ in $\mathrm{H}$ and

$W_{n}(x)=V(x) \cup\left\{a^{i} \mid i \geq n\right.$ and $\left.b^{i} \in V(x)\right\}$.

A family of all sets $W_{n}(x)$ forms completely a system of neighborhoods of $\mathrm{x}$ in $\mathrm{S}$.

\section{Definition 7.}

i) A semiring $\langle S,+, *\rangle$ is a topological semirng if $\mathrm{S}$ is a Hausdorff topological space and both operations, addition "+" and multiplication "*" are continuous.

ii) $\mathrm{A}$ topological semiring $\mathrm{S}$ is a (multiplicatively) monothetic semiring if there exists a generating element $a \in S$ such that $S=\overline{(a)}$.

\section{BASIC LEMMAS}

Now, we will state and prove the following lemmas:

Lemma 1. Let $S$ be a topological semiring, and $R$ be a subsemiring of $\mathrm{S}$, then $\bar{R}$ is a subsemiring of $\mathrm{S}$.

Proof. Assumed that exists two elements $\mathrm{x}, \mathrm{y} \in \overline{\mathrm{R}}$ and $\mathrm{xy}=$ $\mathrm{z} \notin \overline{\mathrm{R}}$. Let $\mathrm{V}(\mathrm{x}), \mathrm{V}(\mathrm{y}), \mathrm{V}(\mathrm{z})$ be neighborhoods such that $\mathrm{V}(\mathrm{x}) \mathrm{V}(\mathrm{y}) \subseteq \mathrm{V}(\mathrm{z})$ and $\mathrm{V}(\mathrm{z}) \cap \overline{\mathrm{R}}=\varnothing$. But exists, $\mathrm{x}_{1} \in \mathrm{R} \cap \mathrm{V}(\mathrm{x})$, $\mathrm{y}_{1} \in \mathrm{R} \cap \mathrm{V}(\mathrm{y})$ and, $\mathrm{x}_{1} \mathrm{y}_{1} \in \mathrm{R} \cap \mathrm{V}(\mathrm{z})$. Thus, we received a contradiction. (The proof about addition is similar).

Lemma 2. Let $S$ be a topological semiring and $R$ an idempotent subsemiring of $\mathrm{S}$, then, $\bar{R}$ is an idempotent subsemiring of $\mathrm{S}$.

Proof. By Lemma 1, $\bar{R}$ is a subsemiring of S. Now, we will suppose that $\mathrm{R}$ is an idempotent subsemiring of $\mathrm{S}$, and that exists $\mathrm{x} \in \bar{R}$ such that $\mathrm{x}+\mathrm{x}=\mathrm{y} \neq \mathrm{x}$. Therefore, there exists the neighborhoods $\mathrm{V}(\mathrm{x}), \mathrm{V}(\mathrm{y})$ such that $\mathrm{V}(\mathrm{x}) \cap \mathrm{V}(\mathrm{y})=\varnothing$ and $\mathrm{V}(\mathrm{x})+\mathrm{V}(\mathrm{x}) \subseteq \mathrm{V}(\mathrm{y})$. Thus, lets $\mathrm{x}_{1} \in \mathrm{R} \cap \mathrm{V}(\mathrm{x})$, then, $\mathrm{x}_{1}+\mathrm{x}_{1}=\mathrm{x}_{1} \in \mathrm{V}(\mathrm{x}) \cap \mathrm{V}(\mathrm{y})$, which lead us to a contradiction.

Lemma 3. Let $\mathrm{S}$ be a topological semiring, and $\mathrm{R}$ be a commutative (relatively to addition) subsemiring of $\mathrm{S}$. Then, $\bar{R}$ is a commutative (relatively to addition) subsemiring of S.

Proof. Let $\mathrm{S}$ be a topological semiring, and $\mathrm{R}$ be a commutative (relatively to addition) subsemiring of S. By Lemma $1, \bar{R}$ is a subsemiring of $\mathrm{S}$. Now, supposed that exists $\mathrm{x}, \mathrm{y} \in \bar{R}$ such that $\mathrm{x}+\mathrm{y} \neq \mathrm{y}+\mathrm{x}$. Let be $\mathrm{x}+\mathrm{y}=\mathrm{z}_{1}$ and $y+x=z_{2}$, with $z_{1} \neq z_{2}$. Let $V(x), V(y), V\left(z_{1}\right), V\left(z_{2}\right)$ be the neighborhoods of $x, y, z_{1}, z_{2}$, respectively such that $\mathrm{V}\left(\mathrm{z}_{1}\right) \cap \mathrm{V}\left(\mathrm{z}_{2}\right)=\varnothing$;

$\mathrm{V}(\mathrm{x})+\mathrm{V}(\mathrm{y}) \subseteq \mathrm{V}\left(\mathrm{z}_{1}\right) ; \mathrm{V}(\mathrm{y})+\mathrm{V}(\mathrm{x}) \subseteq \mathrm{V}\left(\mathrm{z}_{2}\right)$. So, there exists, $\mathrm{x}_{1} \in \mathrm{R} \cap \mathrm{V}(\mathrm{x}) ; \quad \mathrm{x}_{2} \in \mathrm{R} \cap \mathrm{V}(\mathrm{y}) ; \quad \mathrm{x}_{1}+\mathrm{x}_{2}=\mathrm{x}_{2}+\mathrm{x}_{1} \in \mathrm{V}\left(\mathrm{z}_{1}\right) \cap \mathrm{V}\left(\mathrm{z}_{2}\right)$ Therefore, we receive a contradiction.

Lemma 4. Let $S$ be a topological semiring and $R$ be an idempotent subsemiring of S, with left (right) addition, then $\bar{R}$ is an idempotent subsemiring of $\mathrm{S}$ with left (right) addition.

Proof. By Lemma 2, $\bar{R}$ is an idempotent subsemiring of S. And supposed that $\mathrm{R}$ has left addition, let $\mathrm{x}, \mathrm{y}, \mathrm{z} \in \bar{R}$ and $\mathrm{x}+\mathrm{y}=\mathrm{z} \neq \mathrm{x}$. Thus, there exists $\mathrm{V}(\mathrm{x}), \quad \mathrm{V}(\mathrm{y}), \quad \mathrm{V}(\mathrm{z})$ the neighborhoods such that, $\mathrm{V}(\mathrm{x})+\mathrm{V}(\mathrm{y}) \subseteq \mathrm{V}(\mathrm{z})$ and $\mathrm{V}(\mathrm{x}) \cap \mathrm{V}(\mathrm{z})=$ Ø. Let $\quad x_{1} \in R \cap V(x), \quad y_{1} \in R \cap V(y), \quad$ then, $\mathrm{x}_{1}+\mathrm{y}_{1}=\mathrm{x}_{1} \in \mathrm{V}(\mathrm{x}) \cap \mathrm{V}(\mathrm{z})$; which is a contradiction. (The case with right addition can also be proved similarly).

Lemma 5. Let $\mathrm{S}$ be a topological semiring and $\mathrm{R}$ be a commutative (relatively to addition) idempotent subsemiring of $S$, where $\langle R,+\rangle$ is a chain relatively the order $\mathrm{x} \leq \mathrm{y} \Leftrightarrow \mathrm{x}+\mathrm{y}=\mathrm{y}$ for all $\mathrm{x}, \mathrm{y}$ of $\mathrm{S}$. Then, $\langle\bar{R},+>$, is a chain relatively to the order, $x \leq y \Leftrightarrow x+y=y$, for all, $x, y$ of $S$.

Proof. By Lemmas 2 and 3, R* is a commutative (relatively to addition) idempotent subsemiring of S. So, we shall prove that $\bar{R}$ is a chain relatively to the order, $\mathrm{x} \leq \mathrm{y} \Leftrightarrow \mathrm{x}+\mathrm{y}=\mathrm{y}$, for all, $\mathrm{x}, \mathrm{y}$ of $\mathrm{S}$. Supposed that $\mathrm{x}, \mathrm{y}, \mathrm{z} \in \bar{R}$ and $\mathrm{x}+\mathrm{y}=\mathrm{z} \neq \mathrm{x}$, $\mathrm{z} \neq \mathrm{y}, \quad \mathrm{x} \neq \mathrm{y}$. There exists $\mathrm{V}(\mathrm{x}), \mathrm{V}(\mathrm{y})$ and $\mathrm{V}(\mathrm{z})$ the neighborhoods, such that, $\mathrm{V}(\mathrm{x})+\mathrm{V}(\mathrm{y}) \subseteq \mathrm{V}(\mathrm{z})$ and $\mathrm{V}(\mathrm{x}) \cap \mathrm{V}(\mathrm{z})=\mathrm{V}(\mathrm{y}) \cap \mathrm{V}(\mathrm{z})=\varnothing$. So, there exists $\mathrm{x}_{1} \in \mathrm{R} \cap \mathrm{V}(\mathrm{x})$, $\mathrm{y}_{1} \in \mathrm{R} \cap \mathrm{V}(\mathrm{y})$ and $\mathrm{x}_{1}+\mathrm{y}_{1}=\mathrm{x}_{1}$ or $\mathrm{x}_{1}+\mathrm{y}_{1}=\mathrm{y}_{1}$. There, we get to a contradiction, which, means that, $\langle\bar{R},+>$ is a chain relatively to the order $x \leq y \Leftrightarrow x+y=y$, for all $x$, $y$ of $S$. 


\section{MAIN RESULTS}

From Lemmas 4 and 5 and Theorems 1 and 2, follows Theorem 4, as the description of a compact monothetic semiring. This result was announced in [12].

Theorem 4. Let $\mathrm{S}=\langle\mathrm{S},+, *\rangle$, be a compact monothetic semiring. Then be realized one of the following two cases:

i) $\mathrm{S}$ is a discrete finite cyclic semiring;

ii) $\langle$ S, *>, is a non-discrete compact monothetic semigroup and, $\langle\mathrm{S},+\rangle$, or has left addition $(\mathrm{x}+\mathrm{y}=\mathrm{x}$, for all, $\mathrm{x}, \mathrm{y}$ of $\mathrm{S})$, or has right addition $(x+y=y$, for all, $x, y$ of $S)$ or, $\langle S,+\rangle$, is a chain at the relation of order, $x \leq y \Leftrightarrow x+y=y$, for all $x, y$ of $S$.

By the theorem well-known how alternative of Pontryagin a locally compact monothetic group is a discrete or compact [9].

The question about alternative of Pontryagin for locally compact monothetic semigroups has been open for a long time. For example, the author formulated this problem in [10].

In [11], it is presented the example of a non-discrete and a non-compact locally compact monothetic semigroup. And, if we introduce in this semigroup the left addition, it will be transformed into a non-discrete and a non-compact locally compact monothetic semiring. Therefore, also we have theorem 5 .

Theorem 5. There exists non-discrete and non-compact locally compact monothetic semirings.

\section{CONCLUSION}

In the future, we should begin to study so-called singlegenerated semirings. Such are, for example, the factorsemirings of semiring of polynomials in one variable with non-negative integer coefficients.

\section{REFERENCES}

[1] Dedekind R. Uber die Theorie der granzen algebraiscen Zahlen; Supplement XI P.G.

Lejeune Dirichlet: Vorlesung uber Zahlentheorie 4 Aulf, Druck And Verlag,
Braunschweig, 1894.

[2] Vandiver H. S. Note on a simple type of algebra in which cancellation law of addition

does not hold; Bulletin of American Mathematics Society, 1934, Vol. 40, pp 914-920.

[3] Kleene S. C. Representation of events in nerve nets and finite automata; In C. E. Shannon

And McCarthy, editors, Automata Studies, volume 34 of Annals of Mathematics Studies,

p. 3-41, Princeton University Press, Princeton, 1956.

[4] Kuich W. and Salomaa A. Semieings, Automata, Languages, Volume 5 of EATCS

Monographs on Theoretical Computer Science. Springer Berlin, 1986.

[5] Vechtomov E. M., Bestyjev A. S. Cyclic Semirings with commutative addition. Vestnik Syktyvkarskogo Universiteta, Ser 1, Vol 1 (20), 2015, pp. 8-39 (in Russian)

[6] Vechtomov E. M. Lubuagina I. V., Cyclic semirings with noncommutative addition,

Fundamental and Applied Mathematics, Vol. 17, (2011/2012) N 1, pp. 33-52 (in Russian)

[7] Vechtomov E.M. Introduction in Semirings, Kirov, 2000 (in Russian).

[8] Hewitt E. Compact monothetic semigroups. Duke Math. J., 23, N3, 1956, pp. 447 - 457.

[9] Pontryagin L.S. Topological Groups, CRC Press, 1982.

[10] Tanana B.P. Shevrin L.N. Topological Semigroup. In Enciclopedia of Matematics,

Moscow: Sovetckaia Enciclopedia , Volume 5, 1985, pp. 372 - 374 (in Russian).

[11] Zelenyk, E. G. About alternative of Pontryagin for topological semigroups. Matematicheskie Zametki, T. 44, N 3, 1988 c. 402 -403 (in Russian).

[12] Boris Tanana, Bhangy Cassy and Sergei Silvestrov. About Compact Monothetic Semirings. Booklet of abstracts SPAS 2019, $2^{\text {nd }}$ International Conference on Stochastic Processes and Algebraic Structures. Vasteras, Sweden, September 30 - October 2, 2019 pp. 125 - 126.

\begin{abstract}
Author Info
Boris Tanana. Achieved his Ph.D degree in 1980 in Physical Mathematical Sciences. Specialty: Algebra, Mathematical Logic and Theory of Numbers. He was employed at Ural's State University (URGU) in Russia, then at Eduardo Mondlane University and Higher Institute for Science and Technology of Mozambique (ISCTEM), both, in Maputo, Mozambique as Associate Professor. He has more than 60 scientific papers published. His areas of research interest are Topological Algebra Theory of Formal Languages and Mathematics Education.
\end{abstract}

